\title{
Diabetes and NAFLD: a high-risk cohort with definite therapeutic potential
}

\author{
Lucia Brodosi ${ }^{1,2}$, Alessandra Musio ${ }^{1,2}$, Francesca Alessandra Barbanti ${ }^{1,2}$, Dorina Mita ${ }^{1,2}$, Giulio Marchesini ${ }^{1}$, \\ Maria Letizia Petroni ${ }^{1,2}$ \\ 'Department of Medical and Surgical Sciences, "Alma Mater" University, Bologna 40138, Italy. \\ ${ }^{2}$ Azienda Ospedaliero-Universitaria di Bologna, Bologna 40138, Italy.
}

Correspondence to: Dr. Lucia Brodosi, Azienda Ospedaliero-Universitaria di Bologna, via Albertoni 15, Bologna 40138, Italy. E-mail: lucia.brodosi2@unibo.it

How to cite this article: Brodosi L, Musio A, Barbanti FA, Mita D, Marchesini G, Petroni ML. Diabetes and NAFLD: a high-risk cohort with definite therapeutic potential. Hepatoma Res 2020;6:82. http://dx.doi.org/10.20517/2394-5079.2020.88

Received: 21 Aug 2020 First Decision: 23 Sep 2020 Revised: 30 Sep 2020 Accepted: 14 Oct 2020 Published: 5 Dec 2020

Academic Editor: Stefano Bellentani Copy Editor: Cai-Hong Wang Production Editor: Jing Yu

\begin{abstract}
Despite the fact that non-alcoholic fatty liver disease (NAFLD) and its severe clinical forms [non-alcoholic steatohepatitis (NASH) and NASH-cirrhosis] are highly prevalent in the general population, there are no licensed drugs for NAFLD, and lifestyle intervention remains the only treatment accepted by international guidelines. This is despite massive investments in research by pharmaceutical companies. In the presence of type 2 diabetes, novel anti-diabetic drugs offer an opportunity to reduce the burden of NAFLD, by adequate control of glucose and lipid metabolism, also reducing the risk of NASH progression, advanced fibrosis, and finally hepatocellular carcinoma. We extensively reviewed the literature, based either on registration studies, ad hoc randomized studies or real-world data, to define the effectiveness of anti-diabetic drugs in the treatment of NAFLD and prevention of hepatocellular carcinoma (HCC). Metformin provides the best evidence for decreased risk of HCC, pioglitazone was associated with decreased progression to fibrosis, glucagon-like peptide-1 receptor agonists offer a possible opportunity to reduce NAFLD progression coupled with a definite protection for cardiovascular outcomes, and sodium-glucose cotransporter-2 inhibitors are likely to reduce lipid burden, simultaneously reducing the risk of progressive renal and heart failure. For the latter two drug classes, the effects on NAFLD might largely explained by decreased body weight, in keeping with the beneficial effects of intensive lifestyle intervention.
\end{abstract}

Keywords: Metformin, pioglitazone, incretins, DPP-4 inhibitors, GLP-1 receptor agonists, SGLT-2 inhibitors, insulin, cirrhosis 


\section{INTRODUCTION}

The clinical and economic burden associated with non-alcoholic fatty liver disease (NAFLD) is becoming of paramount importance for national health systems globally. Most recent data indicate that approximately $25 \%$ of adults may be classified as NAFLD ${ }^{[1]}$, one in 4 to 5 patients with NAFLD have non-alcoholic steatohepatitis $(\mathrm{NASH})^{[1]}$, and $1.5 \%$ have advanced fibrosis ${ }^{[2]}$, the hallmark of disease progression to cirrhosis $^{[3]}$.

The hepatic disease is part of a multifaced involvement of other tissues and organs, primarily the cardiovascular system and the kidney ${ }^{[4]}$, within the frame of the metabolic syndrome ${ }^{[5]}$, that adds to the liver in driving long-term outcomes ${ }^{[6]}$. For this reason, there is a compelling need to adjust treatment to minimize cardiovascular risk in all patients with NAFLD, as suggested by national ${ }^{[7]}$ and international ${ }^{[8]}$ guidelines.

Despite much research and investment by pharmaceutical companies, no drugs have so far been approved for treatment by regulatory authorities, and adherence to healthier lifestyle remains the only accepted treatment strategy ${ }^{[9]}$. Several drugs failed the agreed treatment outcomes (reduced fibrosis without worsening of NASH or reduced necroinflammation, no worsening of fibrosis ${ }^{[10]}$ ) for approval during phase 2 or phase 3 randomized controlled studies $(\mathrm{RCTs})^{[11]}$; only obeticholic acid fulfilled the targets in a phase 3 study $^{[12]}$, but the Food and Drug Administration required additional studies considering the low benefit/ risk ratio ${ }^{[13]}$.

Individuals with Type 2 diabetes mellitus (T2DM) constitute a large cohort of NAFLD cases. The prevalence of NAFLD in T2DM is as high as $60 \%{ }^{[14]}$, and T2DM increases the risk of disease progression to cirrhosis as well as the occurrence of hepatocellular carcinoma (HCC $)^{[15-17]}$. The relationship between T2DM and NAFLD appears to be bidirectional, with T2DM increasing the risk of NAFLD and NAFLD favoring the development of altered glucose regulation and $\mathrm{T}_{2} \mathrm{DM}^{[18]}$. Initially considered the hepatic manifestation of metabolic syndrome ${ }^{[19]}$, and consequently as a likely effect of diabetes ${ }^{[20]}$, it has also been suggested that liver fat accumulation and NAFLD might indeed be the metabolic driver of $\mathrm{T} 2 \mathrm{DM}^{[21]}$. This evidence makes the development of T2DM an additional outcome of NAFLD treatment and prompts the need for strict control of glucose metabolism in NAFLD cases.

In the past 15 years the treatment of T2DM has completely changed. Second-generation sulfonylureas and glinides, very effective oral drugs long considered the standard of treatment before prescribing insulin injection, have been moved as third-line treatment and limited to rare settings in most recent international guidelines, because of poor durability and a high risk of hypoglycemia and coronary artery disease ${ }^{[22-24]}$. Very effective and safer drugs dipeptidylpeptidase-4 inhibitors (DPP-4Is), glucagon-like peptide-1 receptor agonists [(GLP-1Ras) and sodium-glucose transporter-2 inhibitors (SGLT-2Is)] were added to the classical armamentarium (metformin, acarbose, sulfonylureas and glinides, insulin) with definite advantages on the impending risk of hypoglycemia, cardiovascular disease and heart failure ${ }^{[22]}$. Their efficacy and safety has been demonstrated in registration studies as well as in large cardiovascular outcome trials (CVOTs) required by regulatory agencies such as the FDA and European Medicines Agency ${ }^{[2]}$. The effects on liver fat accumulation have been tested $v s$. sulfonylureas/glinides, or are under investigation. Also, pioglitazone, an insulin-sensitizer of limited use following a series of warning data on class safety, initially involving rosiglitazone $e^{[26]}$, has shown positive effects on cardiovascular outcomes ${ }^{[27]}$. In patients with NAFLD, irrespective of the presence of T2DM, is was associated a reduced risk of advanced fibrosis ${ }^{[28]}$, and its use is now recommended by national and international guidelines ${ }^{[8]}$.

The present review is aimed at defining the role of novel anti-diabetic drugs for the treatment of NAFLD in patients with T2DM, with particular reference to the prevention of HCC. Data were retrieved from ad 
Table 1. Metabolic and clinical effects of anti-diabetic drugs

\begin{tabular}{|c|c|c|c|c|c|}
\hline Drug class & Metabolic control & Hypo-glycemia & $\begin{array}{c}\text { Cardiovascular } \\
\text { system }\end{array}$ & Heart failure & $\begin{array}{c}\text { Specific beneficial/adverse } \\
\text { effects }\end{array}$ \\
\hline Metformin & + & \pm & Uncertain protection & Null & $\begin{array}{l}\text { Cancer protection Acidosis, } \\
\text { anemia }\end{array}$ \\
\hline$\alpha$-Glucosidase inhibitors & + & - & Null & Null & $\begin{array}{l}\text { Modest weight loss GI } \\
\text { discomfort }\end{array}$ \\
\hline Pioglitazone & ++ & \pm & Protective & Increased risk & $\begin{array}{l}\text { Weight gain, non- } \\
\text { osteoporotic fractures }\end{array}$ \\
\hline Sulfonylureas/Glinides & +++ & +++ & Increased risk & Increased risk & $\begin{array}{l}\text { Alcohol interaction Weight } \\
\text { gain, low durability }\end{array}$ \\
\hline DPP-4 inhibitors & ++ & - & Null & Null & $\begin{array}{l}\text { High durability Flu-like } \\
\text { symptoms, runny nose }\end{array}$ \\
\hline GLP-1 receptor agonists & +++ & - & Protective & Null & Weight loss Nausea, vomiting \\
\hline SGLT-2 inhibitors & +++ & - & Protective & Protective & $\begin{array}{l}\text { Weight loss, renal protection } \\
\text { Genito-urinary infections }\end{array}$ \\
\hline $\begin{array}{l}\text { Insulin (basal or basal- } \\
\text { bolus) }\end{array}$ & ++++ & ++++ & Protective $^{\star}$ & Protective $^{\star}$ & $\begin{array}{l}\text { Weight gain, highly negative } \\
\text { impact on quality of life }\end{array}$ \\
\hline
\end{tabular}

*Protection exerted by improved metabolic control; Null: no evidence of specific protection

hoc RCTs, as well as from the re-analysis of large registration or CVOT trials. More recently, several large epidemiological surveys of real-world data became available, and their support to define the best treatment to prevent liver disease progression is also reported.

\section{DATA SEARCH AND ANALYSIS}

We searched PubMed and www.clinicaltrials.gov for studies on novel anti-diabetic drug use in patients with NAFLD or NASH. In PubMed we used the string [liver steatosis (MeSH Terms)] OR [NAFLD (MeSH Terms)] OR [HCC (Text Word)] OR [carcinoma (Text Word)] AND [adult onset diabetes mellitus (MeSH Terms)] AND [treatment (Text Word)] filtered by "humans". The string retrieved 694 references published in the period 1988-2020. On www.clinicaltrials.gov we used the string "NAFLD OR NASH" as "condition or disease" field, while the names of the classes and later the names of the individual molecules were entered in the "other terms" field. On the left bar, in "Study Phase" we selected "Phase 2, 3, and 4". Only studies with more than 10 participants were considered. Later, the references of all retrieved studies and review articles were scrutinized for missing references, and duplicate studies were removed. Data of the available evidence is summarized in Table 1.

\section{RESULTS}

\section{Metformin}

Metformin has long been considered the first-line drug for the treatment of T2DM and it is still indicated for all individuals who can tolerate its use without gastrointestinal discomfort. Despite its insulinsensitizing activity, potentially reducing lipid burden, metformin is no longer specifically indicated for NAFLD, following a few studies and a review article where it failed to reduce histological severity of NAFLD $^{[29]}$. However, metformin is now living a second life, as it were, considering its HCC-preventive action $^{[30-32]}$, coupled with reduced all-site cancer risk ${ }^{[33]}$. Continuation of metformin was also shown to improve overall survival in NASH-cirrhosis with Child-Pugh class A and $\mathrm{B}^{[34]}$; these beneficial effects justify the statement of international guidelines suggesting the use of background metformin for all T2DM patients with $\mathrm{NAFLD}^{[8]}$.

\section{Pioglitazone}

Pioglitazone is an anti-diabetic drug that activates peroxisome proliferator-activated receptor- $\gamma$ (PPAR $\gamma$ ), a nuclear receptor, mostly expressed in the adipose tissue, and to a lesser extent in other organs, including the liver. The activation of the PPAR $\gamma$ quells the production of liver collagen by hepatic stellate cells, 
promotes the differentiation of adipocytes, decreases leptin and IL-6 concentration, increases adiponectin levels, and above all, reduces insulin resistance, the driver of $\mathrm{NAFLD}^{[35]}$. Pioglitazone has been tested in NAFLD at the target dose of 30-45 mg/day in several RCTs with histologic outcomes, showing a reduction of necroinflammation (NAFLD activity score - NAS) ${ }^{[36-40]}$, as well as improvement in fibrosis in a systematic meta-analysis ${ }^{[41]}$. Pioglitazone also reduces the risk of cardiovascular and cerebrovascular outcomes ${ }^{[22,43]}$, as well as of HCC (odds ratio, OR $=0.83,95 \% \mathrm{CI}$ : 0.72-0.95) ${ }^{[44]}$. This makes pioglitazone the treatment of choice of NASH, independent of the presence of T2DM. Notably, treatment discontinuation is followed by NASH recurrence ${ }^{[45]}$. Pioglitazone treatment is associated with moderate weight gain, and the risks of non-osteoporotic fractures and, particularly, of heart failure are also increased; for these reasons the drug should not be used in elderly patients ${ }^{[46]}$. Adverse events are probably rare at lower doses $(15 \mathrm{mg} /$ day), but the effects on the liver are also unknown. At present, the use of pioglitazone is off-label outside T2DM and informed consent is needed before treatment in individuals without diabetes.

\section{Dipeptidyl-peptidase-4 inhibitors}

DPP-4Is (sitagliptin, vildagliptin, saxagliptin and alogliptin) decrease blood glucose by preventing the rapid degradation in incretins, thus increasing glucose-dependent insulin release ${ }^{[47]}$. This class of antidiabetic drugs has progressively entered the market in the past 15 years, showing a moderate effect on glucose control, and no risk of hypoglycemia or adverse cardiovascular outcomes. A meta-analysis by Carbone et al. ${ }^{[4]]}$ on the effects of incretin treatment in patients with NASH and T2DM including 66 participants treated with sitagliptin for between 16 and 36 weeks found a significant mean reduction of alanine aminotransferase (ALT) in the two sitagliptin-treated cohorts (mean 17.7 U/L; 95\%CI: 12.4-23.1; $P<0.001)$. In a small cohort with T2DM and NASH, the administration of sitagliptin $100 \mathrm{mg} /$ day for one year determined a significant improvement in hepatocyte ballooning $(P=0.014)$ and total NAS $(P=0.04)$, as well as a decrease in ALT and aspartate aminotransferase (AST), an index more closely correlated with chronic liver damage ${ }^{[49]}$. Similar data on liver enzymes were reported in 44 patients treated for six months with DPP-4Is ${ }^{[50]}$, whereas the improvement of NAS was confirmed in 40 NASH patients ${ }^{[51]}$, randomized to lifestyle changes $v s$. lifestyle changes associated with sitagliptin (NAS: $-1.9 \pm 1.4$ vs. $-0.7 \pm 1.1 ; P=0.006$ ).

On the contrary, no differences in aminotransferases, liver fat content or liver stiffness were reported in a 24 -week RCT including patients with pre-diabetes or early diabetes ${ }^{[52,53]}$, treated with sitagliptin (100 mg per day), as well as in two studies in which sitagliptin was tested against placebo for 12 weeks (no differences in serum liver enzymes, hepatic fat content, fibrosis). No differences were reported in surrogate biomarkers of fibrosis, namely NAFLD fibrosis score [NFS], Fibrosis-4 score [FIB-4], aminotransferase-toplatelet ratio index $[\mathrm{APRI}]^{[51,53]}$. In summary, the use of DPP-4Is in T2DM with NAFLD appears to be safe, but without any systematic advantage on progressive liver disease. There are no specific studies on their possible effects on the risk of HCC in T2DM.

\section{Glucagon-like petide-1 receptor agonists}

GLP-1RAs (exenatide, lixisenatide, liraglutide, dulaglutide, semaglutide) are potent injectable anti-diabetic drugs, mimicking the effects of endogenous incretins on insulin release, gastrointestinal motility, and the central nervous system (reduced appetite and food intake ${ }^{[47]}$ ). CVOTs demonstrated that GLP-1RAs, as a class but with some differences between rapid- (exenatide b.i.d. and lixisenatide) and long-acting drugs, reduce the risk of major cardiovascular events in $\mathrm{T}_{2} \mathrm{DM}^{[23]}$, and lead to a systematic weight loss ${ }^{[54]}$. In patients with NAFLD and T2DM, liraglutide was initially reported to reduce liver inflammation (AST, ALT) and liver fibrosis scores (APRI index). These favorable effects might possibly derive from or be enhanced by the concomitant weight and $\mathrm{HbA} 1 \mathrm{c}$ reduction ${ }^{[55]}$. Eguchi et al. ${ }^{[56]}$ also found a reduction in NAS and Brunt's classification grade after a 96-week treatment with liraglutide in ten patients with biopsy-proven NASH/ NAFLD. 
A beneficial role of liraglutide has been convincingly demonstrated in the pilot LEAN (Liraglutide Efficacy and Action in NASH) study ${ }^{[57]}$, a 48 -week RCT in which liraglutide was tested $v s$. placebo in 52 patients with biopsy-confirmed NASH. The study included patients with stage 3 fibrosis (38\% in liraglutide vs. $8 \%$ in placebo) and cirrhosis ( $8 \% v s .15 \%$, respectively), and $35 \%$ of the liraglutide group had T2DM (vs. $31 \%$ in placebo). Liraglutide led to histologic NASH resolution in $35 \%$ of cases, compared with $8 \%$ of placebo-treated patients [relative risk (RR) 4.5; 95\%CI: 1.1-18.9; $P=0.017$ ]. Specifically, liraglutide led to the resolution of NASH in 3 out of 8 patients with T2DM (38\%) (RR $=4.7,95 \% \mathrm{CI}: 0.3-75, P=0.020)$, and only $9 \%$ of patients in the liraglutide group $v s .36 \%$ in the placebo group had fibrosis progression during treatment.

Less convincing data support a similar role for dulaglutide. A post-hoc analysis of the phase 3 AWARD studies [Assessment of Weekly AdministRation of LY2189265 (Dulaglutide) in Diabetes], involving 760 patients with T2DM and high likelihood of NAFLD/NASH based on elevated ALT values and exclusion of other hepatic diseases, showed a significantly greater reduction of ALT after 6-month treatment with dulaglutide $1.5 \mathrm{mg}$ once a week $(-2.1 \mathrm{IU} / \mathrm{L} ; 95 \% \mathrm{CI}$ : -3.9 to $-0.3 ; P=0.022)$. Similar changes were observed when the results were adjusted for body weight $(-8.7 \mathrm{IU} / \mathrm{L} ; 95 \% \mathrm{CI}:-10.1 \text { to }-7.3)^{[58]}$.

Exenatide also reduced ALT and AST levels in people with T2DM and elevated baseline ALT levels ${ }^{[59]}$ in a case series of eight patients with NASH treated for 28 weeks. Some patients also experienced an improvement in histological features, including fibrosis ${ }^{[60]}$. Furthermore, the previously mentioned metaanalysis by Carbone et al. ${ }^{[48]}$ showed a significant mean ALT reduction in both the liraglutide and exenatide treated cohorts (mean 12.2 U/L; 95\%CI: 4.9-19.4; $P<0.001$ ). Finally, exenatide effectively reduced hepatic triglyceride content compared to reference treatment $(+12.5 \pm 9.6 \%, P=0.007$, when assigned to 44 obese subjects with $\mathrm{T} 2 \mathrm{DM}^{[61]}$, again in a weight loss-dependent manner; $\left.r=0.47, P=0.03\right)$. Cuthbertson et al. ${ }^{[62]}$ reported a $42 \%$ median reduction of intracellular fat content $(P<0.0001)$, measured by magnetic resonance spectroscopy (MRS), independently of weight loss, after six months of exenatide or liraglutide.

GLP-1RAs have also been investigated in combination with lifestyle interventions or other drugs. Fan et al. ${ }^{[63]}$ found a significant reduction in ALT, AST, and gamma-glutamyl transpeptidase, and an increase in the AST/ALT ratio in a cohort of 49 patients affected by both T2DM and NAFLD and treated by the combination of exenatide and lifestyle interventions. The MRS-assessed hepatic content was significantly higher in individuals receiving the combination of exenatide and pioglitazone for 12 months (12.1 \pm 1.7 to 4.7 $\pm 1.3 \%)$, however, compared with pioglitazone alone $(11.0 \pm 3.1 \text { to } 6.5 \pm 1.9 \%)^{[64]}$.

A phase 2 study of semaglutide, a longer-acting, weekly dosing GLP-1 analogue, has recently been completed. A preliminary release reports that after 72 weeks of therapy with the highest dosage tested $(0.4 \mathrm{mg})$, 33 of 56 patients (59\%) with fibrosis stages F2 to F3 met the primary end-point of NASH resolution and no worsening in liver fibrosis, $v s .10$ of 58 patients $(17 \%)$ in the control arm $^{[65]}$. Semaglutide is very effective on body weight; a phase 3-4 trial in obesity reported a mean weight loss of $14.9 \%$ with semaglutide $2.4 \mathrm{mg} / \mathrm{week}$ for 68 weeks, increasing to $17.4 \%$ at follow up ${ }^{[66]}$. An oral formulation of semaglutide is also being tested in pre-registration studies ${ }^{[6]]}$.

Concern on the use of GLP-1RA in NASH cirrhosis was recently raised by the observation that liraglutide. While providing optimal control of blood glucose, $\mathrm{HbA1c}$, and body weight in patients, it blunted the effect of beta-blockers on heart rate, possibly indicating a raised bleeding risk after starting GLP-1 RA ${ }^{[68]}$. The researchers proposed a mechanistic molecular explanation of how a GLP-1RA might prevent betaadrenergic receptor blockade ${ }^{[69]}$. For this reason, the treatment of T2DM with GLP-1RA in subjects at risk of bleeding requires additional studies. 


\section{Sodium-glucose co-transporter-2 inhibitors (Gliflozins)}

Empagliflozin, dapagliflozin, canagliflozin, ertugliflozin, and many other SGLT-2Is under development block renal exchange of glucose in the proximal tubule, being responsible for the reuptake of $90 \%$ of the pre-urinary glucose $\mathrm{e}^{[70]}$. They entered the market in the last decade; registration and CVOT trials showed that gliflozins reduce cardiovascular events and, particularly, heart failure ${ }^{[71]}$, prevent the deterioration of renal function ${ }^{[72]}$, and induce a moderate weight $\operatorname{loss}^{[73]}$. The risk of genitourinary tract infections are the principal adverse events associated with gliflozin use ${ }^{[74]}$.

Their effects of SGLT-2Is on liver fat have not been systematically studied, but a few data have recently become available, based either on RCTs or epidemiological studies. In a RCT involving 84 patients, dapagliflozin significantly reduced hepatic fat content measured by magnetic resonance imaging (dapagliflozin, from $17.3 \%$ to $15.1 \%, P<0.05$; placebo from $15.1 \%$ to $14.5 \%$, P not significant), as well as liver enzymes (AST, ALT, GGT) when compared to placebo ${ }^{[75]}$. Similar results on liver fat were reported in a prospective RCT with empagliflozin involving 50 patients (mean difference between patients treated with and without empagliflozin, $-4 \% ; P<0.0001)^{[76]}$, and in another RCT in 20 patients treated with canagliflozin (from $17.6 \% \pm 7.5 \%$ to $12.0 \% \pm 4.6 \%$ after 6 months and $12.1 \% \pm 6.1 \%$ after 12 months; $P<0.005$ for both) ${ }^{[77]}$.

In real-world studies, a larger reduction in liver enzymes is commonly observed during treatment with SGLT-2Is when compared with other antidiabetic drugs ${ }^{[78-81]}$, such as sulfonylureas ${ }^{[80]}$ or DPP-4Is ${ }^{[81]}$. In a large observational study involving 3,667 patients with T2DM, after a mean follow-up of 4.8 months, ALT levels (independently of weight and $\mathrm{HbA}_{1 \mathrm{c}}$ ) were lower in the group treated with canagliflozin and dapagliflozin, compared with those treated with liraglutide and sitagliptin ${ }^{[82]}$.

Very few data are available on SGLT-2Is and histological changes in NAFLD patients. In a prospective open-label study involving five patients who underwent serial liver biopsies, all patients treated with canagliflozin had an improvement in liver steatosis and NAS at 24 weeks, together with a decrease in fibrosis stage in two of them ${ }^{[83]}$. The authors also confirmed these results in nine patients after 24 weeks of canagliflozin treatment, with reduced lobular inflammation, ballooning, and fibrosis stage in $33 \%, 22 \%$, and $33 \%$ of patients, respectively ${ }^{[83]}$.

A significant proportion of the beneficial effects of gliflozins might be derived by reduced body weight. A network meta-analysis of 29 RCTs confirmed that gliflozin treatment was significantly associated with a higher probability to achieve significant weight loss $(\geq 5 \%) v s$. placebo ${ }^{[84]}$. In a recent study, canagliflozin was also reported to reduce the risk of prostate, lung, and pancreatic cancers, without deleterious effects on $\mathrm{HCC}^{[85]}$.

\section{CONCLUSION}

Progress in pharmacotherapy of T2DM has opened interesting areas of research and treatment for patients with NAFLD. The use of old drugs should be systematically abandoned in favor of safer and effective treatments, also addressing the associated cardiovascular and cancer risks, as well as the impending risk of hypoglycemia that may be particularly harmful for frail patients with NASH and non-NASH cirrhosis. A decalogue summarizing the novel evidence is reported in Table 2. Needless to say that the use of novel drugs must be accompanied by intense lifestyle interventions, the only effective strategy to reduce the burden of NAFLD in the long term, as well as by adherence to international guidelines, supporting a change from treatment-to-target to treatment-to-cure, while being respectful of patients' frailty and economic resources ${ }^{[86]}$.

Insulin treatment remains the most effective therapy to control glucose metabolism in very advanced stages; the risk of hypoglycemia and insulin-associated lipogenesis and weight gain - as well as difficulties 
1. Implement a systematic, intensive, continuing lifestyle intervention (healthy diet and habitual physical activity) aimed at maintaining or slowly achieving a near-normal body weight. Physical activity is particularly needed to prevent sarcopenia

2. Carefully assess NAFLD stage by surrogate biomarkers, as well as T2DM comorbidities (cardiovascular and renal involvement). In patients with cirrhosis determine Child-Pugh class and MELD score

3. Define treatment targets on the basis of patients' frailty and disease severity. Although $\mathrm{HbA} 1 \mathrm{c}$ below $6.5 \%$ (48 mmol/mol) may be the desired target in subjects without comorbidities, in individual cases values up to $8 \%(64 \mathrm{mmol} / \mathrm{mol}) \mathrm{may}$ be acceptable. Consider that $\mathrm{HbA1c}$ may be unreliable in the presence of recent hemorrhage, and random glucose monitoring may be advisable

4. Background metformin ( $2 \mathrm{~g} /$ day) treatment should always be used and maintained also in compensated cirrhosis, although at reduced doses (1-1.5 g/day), as long as compatible with gastrointestinal symptoms and renal function

5. Sulfonylureas and glinides should not be used, except as third-line therapy; they both increase the risk of hypoglycemia, and sulfonylureas are also associated with increased cardiovascular risk

6. Add pioglitazone (30-45 mg/day) in patients not at risk of heart failure or ascites. Further intensify lifestyle intervention to prevent weight gain

7. Add DPP4-Is to improve glucose control to near-normal glucose targets in patients without comorbidities

8. Add GLP-1RAs in patients at high risk of cardiovascular disease, including patients with previous cardiovascular events. Caution should be used in subjects with cirrhosis at risk of bleeding

9. SGLT2-Is should be preferred in patients at risk of heart failure, as well as in patients with progressive decline of glomerular filtration rate. Consider the risk of genitourinary infection, particularly in women and in elderly men with prostate problems

10. Avoid insulin use as long as possible, to reduce the risk of hypoglycemia and the impact on quality of life. Late insulin use may be needed in most advanced stages; whenever possible use basal or basal-bolus regimens. Combination of basal insulin with GLP1RAs may be a likely option in selected cases

to lose weight for subjects with obesity - suggests that efforts should be aimed at limiting insulin use. The use of oral DPP-4Is and, later, of weekly-injectable GLP-1RAs or SGLT-2Is in comparison to basal insulin is under investigation ${ }^{[87,88]}$. There is evidence that early initiation of GLP-1RAs may achieve similar or even better results than treatment with basal insulin ${ }^{[89,90]}$ and the improved ease of treatment is associated with better quality of life in advanced disease states. Fewer data are available for SGLT-2Is, but also this drug class appears to be non-inferior to add-on basal insulin as to effectiveness and safety ${ }^{[1,92]}$.

In conclusion, we are living a totally new era in the pharmacologic treatment of type 2 diabetes and patients with NAFLD are likely to take the greatest advantage from novel agents. The beneficial effects of GLP-1RAs and SGLT-2Is on metabolic outcomes extend well beyond the area of diabetes, namely to obesity, cardiovascular risk, heart failure and renal disease ${ }^{[93,94]}$, and might soon be available for NAFLD patients outside of $\mathrm{T} 2 \mathrm{DM}^{[95,96]}$.

\section{DECLARATIONS}

\section{Authors' contributions}

Made substantial contributions to conception and design of the study and interpretation: Brodosi L, Musio A, Marchesini G, Petroni ML

Performed data acquisition, as well as provided technical, and material support: Barbanti FA, Mita D

Drafted the manuscript: Brodosi L, Marchesini G

\section{Availability of data and materials}

Not applicable.

\section{Financial support and sponsorship}

Barbanti FA is supported by a contract financed by Italian Ministry of Health and Italian Regions (NET2016-02364191).

\section{Conflicts of interest}

All authors declared that there are no conflicts of interest in relation to the material presented here. 


\section{Ethical approval and consent to participate}

Not applicable.

\section{Consent for publication}

Not applicable.

\section{Copyright}

(c) The Author(s) 2020.

\section{REFERENCES}

1. Younossi ZM, Koenig AB, Abdelatif D, Fazel Y, Henry L, Wymer M. Global epidemiology of nonalcoholic fatty liver disease-Metaanalytic assessment of prevalence, incidence, and outcomes. Hepatology 2016;64:73-84.

2. Petta S, Di Marco V, Pipitone RM, et al. Prevalence and severity of nonalcoholic fatty liver disease by transient elastography: genetic and metabolic risk factors in a general population. Liver Int 2018;38:2060-8.

3. Taylor RS, Taylor RJ, Bayliss S, et al. Association between fibrosis stage and outcomes of patients with nonalcoholic fatty liver disease: a systematic review and meta-analysis. Gastroenterology 2020;158:1611-25.e12.

4. Loria P, Marchesini G, Nascimbeni F, et al. Cardiovascular risk, lipidemic phenotype and steatosis. A comparative analysis of cirrhotic and non-cirrhotic liver disease due to varying etiology. Atherosclerosis 2014;232:99-109.

5. Marchesini G, Babini M. Nonalcoholic fatty liver disease and the metabolic syndrome. Minerva Cardioangiol 2006;54:229-39.

6. Ekstedt M, Franzen LE, Mathiesen UL, Thorelius L, Holmqvist M, et al. Long-term follow-up of patients with NAFLD and elevated liver enzymes. Hepatology 2006;44:865-73.

7. Italian Association for the Study of the Liver (AISF). AISF position paper on nonalcoholic fatty liver disease (NAFLD): updates and future directions. Dig Liver Dis 2017;49:471-83.

8. European Association for the Study of the Liver (EASL), European Association for the Study of Diabetes (EASD), European Association for the Study of Obesity (EASO). EASL-EASD-EASO Clinical Practice Guidelines for the management of non-alcoholic fatty liver disease. J Hepatol 2016;64:1388-402.

9. Sanyal AJ. Past, present and future perspectives in nonalcoholic fatty liver disease. Nat Rev Gastroenterol Hepatol 2019;16:377-86.

10. Sanyal AJ, Brunt EM, Kleiner DE, et al. Endpoints and clinical trial design for nonalcoholic steatohepatitis. Hepatology 2011;54:344-53.

11. Rinella ME, Noureddin M. STELLAR 3 and STELLAR 4: lessons from the fall of Icarus. J Hepatol 2020;73:9-11.

12. Younossi ZM, Ratziu V, Loomba R, et al. Obeticholic acid for the treatment of non-alcoholic steatohepatitis: interim analysis from a multicentre, randomised, placebo-controlled phase 3 trial. Lancet 2019;394:2184-96.

13. Intercept release. Complete Response Letter (CRL) from the FDA regarding our new drug application for obeticholic acid (OCA) for the treatment of liver fibrosis due to NASH. Available from: https:/ir.interceptpharma.com/news-releases/news-release-details/interceptreceives-complete-response-letter-fda-obeticholic-acid;2020. [Last accessed on 22 Oct 2020]

14. Portillo-Sanchez P, Bril F, Maximos M, et al. High prevalence of nonalcoholic fatty liver disease in patients with type 2 diabetes mellitus and normal plasma aminotransferase levels. J Clin Endocrinol Metab 2015;100:2231-8.

15. Hazlehurst JM, Woods C, Marjot T, Cobbold JF, Tomlinson JW. Non-alcoholic fatty liver disease and diabetes. Metabolism 2016;65:1096-108.

16. Hossain N, Afendy A, Stepanova M, et al. Independent predictors of fibrosis in patients with nonalcoholic fatty liver disease. Clin Gastroenterol Hepatol 2009; 7:1224-9, 29.e1-2.

17. Jarvis H, Craig D, Barker R, et al. Metabolic risk factors and incident advanced liver disease in non-alcoholic fatty liver disease (NAFLD): a systematic review and meta-analysis of population-based observational studies. PLoS Med 2020;17:e1003100.

18. Ballestri S, Zona S, Targher G, et al. Nonalcoholic fatty liver disease is associated with an almost twofold increased risk of incident type 2 diabetes and metabolic syndrome. Evidence from a systematic review and meta-analysis. J Gastroenterol Hepatol 2016;31:936-44.

19. Marchesini G, Bugianesi E, Forlani G, et al. Nonalcoholic fatty liver, steatohepatitis, and the metabolic syndrome. Hepatology 2003;37:917-23.

20. Marchesini G, Brizi M, Bianchi G, et al. Nonalcoholic fatty liver disease: a feature of the metabolic syndrome. Diabetes 2001;50:1844-50.

21. Taylor R. Pathogenesis of type 2 diabetes: tracing the reverse route from cure to cause. Diabetologia 2008;51:1781-9.

22. American Diabetes Association. 9. Pharmacologic Approaches to Glycemic Treatment: Standards of Medical Care in Diabetes-2020. Diabetes Care 2020;43:S98-110.

23. Kristensen SL, Rørth R, Jhund PS, et al. Cardiovascular, mortality, and kidney outcomes with GLP-1 receptor agonists in patients with type 2 diabetes: a systematic review and meta-analysis of cardiovascular outcome trials. Lancet Diab Endocrinol 2019;7:776-85.

24. Zelniker TA, Wiviott SD, Raz I, et al. SGLT2 inhibitors for primary and secondary prevention of cardiovascular and renal outcomes in type 2 diabetes: a systematic review and meta-analysis of cardiovascular outcome trials. Lancet 2019;393:31-9.

25. U.S. Department of Health and Human Services FaDA, Center for Drug Evaluation and Research. Guidance for Industry. Diabetes mellitus - evaluating cardiovascular risk in new antidiabetic therapies to treat type 2 diabetes. 2008. Available from: http://wwwfdagov/ downloads/drugs/guidancecomplianceregulatoryinformation/guidances/ucm071627pdf. [Last accessed on 22 Oct 2020]

26. Nissen SE, Wolski K. Effect of rosiglitazone on the risk of myocardial infarction and death from cardiovascular causes. $N$ Engl J Med 
2007;356:2457-71.

27. Lincoff AM, Wolski K, Nicholls SJ, Nissen SE. Pioglitazone and risk of cardiovascular events in patients with type 2 diabetes mellitus: a meta-analysis of randomized trials. JAMA 2007;298:1180-8.

28. Musso G, Cassader M, Paschetta E, Gambino R. Pioglitazone for advanced fibrosis in nonalcoholic steatohepatitis: New evidence, new challenges. Hepatology 2017;65:1058-61.

29. Donadon V, Balbi M, Mas MD, Casarin P, Zanette G. Metformin and reduced risk of hepatocellular carcinoma in diabetic patients with chronic liver disease. Liver Int 2010;30:750-8.

30. Zhang ZJ, Zheng ZJ, Shi R, Su Q, Jiang Q, Kip KE. Metformin for liver cancer prevention in patients with type 2 diabetes: a systematic review and meta-analysis. J Clin Endocrinol Metab 2012;97:2347-53.

31. Singh S, Singh PP, Singh AG, Murad MH, Sanchez W. Anti-diabetic medications and the risk of hepatocellular cancer: a systematic review and meta-analysis. Am J Gastroenterol 2013;108:881-91; quiz 892.

32. Yu H, Zhong X, Gao P, et al. The potential effect of metformin on cancer: an umbrella review. Front Endocrinol (Lausanne) $2019 ; 10: 617$.

33. Zhang X, Harmsen WS, Mettler TA, et al. Continuation of metformin use after a diagnosis of cirrhosis significantly improves survival of patients with diabetes. Hepatology 2014;60:2008-16.

34. Hauner H. The mode of action of thiazolidinediones. Diabetes Metab Res Rev 2002;18:S10-5.

35. Hauner H. The mode of action of thiazolidinediones. Diabetes Metab Res Rev 2002;18:S10-5.

36. Belfort R, Harrison SA, Brown K, Darland C, Finch J, et al. A placebo-controlled trial of pioglitazone in subjects with nonalcoholic steatohepatitis. N Engl J Med 2006;355:2297-307.

37. Belfort R, Harrison SA, Brown K. A placebo-controlled trial of pioglitazone in subjects with nonalcoholic steatohepatitis. N Engl J Med 2006;355:2297-307.

38. Chalasani NP, Sanyal AJ, Kowdley KV, et al. Pioglitazone versus vitamin E versus placebo for the treatment of non-diabetic patients with non-alcoholic steatohepatitis: PIVENS trial design. Contemp Clin Trials 2009;30:88-96.

39. Promrat K, Lutchman G, Uwaifo GI, et al. A pilot study of pioglitazone treatment for nonalcoholic steatohepatitis. Hepatology 2004;39:188-96.

40. Sanyal AJ, Chalasani N, Kowdley KV, et al; NASH CRN. Pioglitazone, vitamin E, or placebo for nonalcoholic steatohepatitis. $N$ Engl $J$ Med 2010;362:1675-85.

41. Musso G, Cassader M, Paschetta E, Gambino R. Thiazolidinediones and advanced liver fibrosis in nonalcoholic steatohepatitis: a metaanalysis. JAMA Intern Med 2017;177:633-40.

42. Dormandy JA, Charbonnel B, Eckland DJ, et al. Secondary prevention of macrovascular events in patients with type 2 diabetes in the PROactive Study (PROspective pioglitAzone Clinical Trial In macroVascular Events): a randomised controlled trial. Lancet 2005;366:1279-89.

43. Kernan WN, Viscoli CM, Furie KL, et al; IRIS Trial Investigators. Pioglitazone after ischemic stroke or transient ischemic attack. $N$ Engl J Med 2016;374:1321-31.

44. Chang CH, Lin JW, Wu LC, Lai MS, Chuang LM, Chan KA. Association of thiazolidinediones with liver cancer and colorectal cancer in type 2 diabetes mellitus. Hepatology 2012;55:1462-72.

45. Bril F, Lomonaco R, Kalavalapalli S, Lai J, Cusi K. 223-OR: Pioglitazone discontinuation in patients with nonalcoholic steatohepatitis (NASH) is associated with disease recurrence. Diabetes 2019;68:223-OR.

46. Motola D, Piccinni C, Biagi C, et al. Cardiovascular, ocular and bone adverse reactions associated with thiazolidinediones: a disproportionality analysis of the US FDA adverse event reporting system database. Drug Saf 2012;35:315-23.

47. Drucker DJ, Nauck MA. The incretin system: glucagon-like peptide-1 receptor agonists and dipeptidyl peptidase-4 inhibitors in type 2 diabetes. Lancet 2006;368:1696-705.

48. Carbone LJ, Angus PW, Yeomans ND. Incretin-based therapies for the treatment of non-alcoholic fatty liver disease: a systematic review and meta-analysis. J Gastroenterol Hepatol 2016;31:23-31.

49. Yilmaz Y, Yonal O, Deyneli O, Celikel CA, Kalayci C, Duman DG. Effects of sitagliptin in diabetic patients with nonalcoholic steatohepatitis. Acta Gastroenterol Belg 2012;75:240-4.

50. Kanazawa I, Tanaka K, Sugimoto T. DPP-4 inhibitors improve liver dysfunction in type 2 diabetes mellitus. Med Sci Monit 2014;20:1662-7.

51. Alam S, Ghosh J, Mustafa G, Kamal M, Ahmad N. Effect of sitagliptin on hepatic histological activity and fibrosis of nonalcoholic steatohepatitis patients: a 1-year randomized control trial. Hepat Med 2018;10:23-31.

52. Cui J, Philo L, Nguyen P, et al. Sitagliptin vs. placebo for non-alcoholic fatty liver disease: a randomized controlled trial. J Hepatol 2016;65:369-76.

53. Smits MM, Tonneijck L, Muskiet MH, et al. Twelve week liraglutide or sitagliptin does not affect hepatic fat in type 2 diabetes: a randomised placebo-controlled trial. Diabetologia 2016;59:2588-93.

54. Brown E, Cuthbertson DJ, Wilding JP. Newer GLP-1 receptor agonists and obesity-diabetes. Peptides 2018;100:61-7.

55. Armstrong MJ, Houlihan DD, Rowe IA, et al. Safety and efficacy of liraglutide in patients with type 2 diabetes and elevated liver enzymes: individual patient data meta-analysis of the LEAD program. Aliment Pharmacol Ther 2013;37:234-42.

56. Eguchi Y, Kitajima Y, Hyogo H, et al; Japan Study Group for NAFLD (JSG-NAFLD). Pilot study of liraglutide effects in non-alcoholic steatohepatitis and non-alcoholic fatty liver disease with glucose intolerance in Japanese patients (LEAN-J). Hepatol Res 2015;45:269-78.

57. Armstrong MJ, Gaunt P, Aithal GP, et al. Liraglutide safety and efficacy in patients with non-alcoholic steatohepatitis (LEAN): a multicentre, double-blind, randomised, placebo-controlled phase 2 study. The Lancet 2016;387:679-90. 
58. Cusi K, Sattar N, García-Pérez LE, et al. Dulaglutide decreases plasma aminotransferases in people with Type 2 diabetes in a pattern consistent with liver fat reduction: a post hoc analysis of the AWARD programme. Diabet Med 2018;35:1434-9.

59. Buse JB, Klonoff DC, Nielsen LL, et al. Metabolic effects of two years of exenatide treatment on diabetes, obesity, and hepatic biomarkers in patients with type 2 diabetes: an interim analysis of data from the open-label, uncontrolled extension of three double-blind, placebo-controlled trials. Clin Ther 2007;29:139-53.

60. Kenny PR, Brady DE, Torres DM, Ragozzino L, Chalasani N, Harrison SA. Exenatide in the treatment of diabetic patients with nonalcoholic steatohepatitis: a case series. Am J Gastroenterol 2010;105:2707-9.

61. Dutour A, Abdesselam I, Ancel P, et al. Exenatide decreases liver fat content and epicardial adipose tissue in patients with obesity and type 2 diabetes: a prospective randomized clinical trial using magnetic resonance imaging and spectroscopy. Diabetes Obes Metab 2016;18:882-91.

62. Cuthbertson DJ, Irwin A, Gardner CJ, et al. Improved glycaemia correlates with liver fat reduction in obese, type 2 diabetes, patients given glucagon-like peptide-1 (GLP-1) receptor agonists. PLoS One 2012;7:e50117.

63. Fan H, Pan Q, Xu Y, Yang X. Exenatide improves type 2 diabetes concomitant with non-alcoholic fatty liver disease. Arq Bras Endocrinol Metabol 2013;57:702-8.

64. Sathyanarayana P, Jogi M, Muthupillai R, Krishnamurthy R, Samson SL, Bajaj M. Effects of combined exenatide and pioglitazone therapy on hepatic fat content in type 2 diabetes. Obesity (Silver Spring) 2011;19:2310-5.

65. NOVO Nordisk. Semaglutide in NASH phase 2 trial successfully completed. Financial report for the period 1 January 2020 to 31 March 2020. Available from: https://www.novonordisk.com/content/dam/Denmark/HQ/investors/irmaterial/quarterly_financial_reports/2020/ Financial\%20report\%20for\%20Q1\%202020.pdf;2020. [Last accessed on 22 Oct 2020]

66. NOVO Nordisk. Semaglutide $2.4 \mathrm{mg}$ demonstrates superior and sustained weight loss versus placebo and in addition a $17.4 \%$ weight loss after 68 weeks in STEP 4 trial. In: editor^editors, editor. GlobeNewswire. Available from: https://ml-eu.globenewswire.com/Resource/ Download/4951d1a2-3bd1-47ea-840a-a1234109c018;2020. [Last accessed on 22 Oct 2020]

67. Zinman B, Aroda VR, Buse JB, et al; PIONEER 8 Investigators. Efficacy, safety, and tolerability of oral semaglutide versus placebo added to insulin with or without metformin in patients with type 2 diabetes: the PIONEER 8 trial. Diabetes Care2019;42:2262-71.

68. Vukotic R, Raimondi F, Brodosi L, et al. The effect of liraglutide on $\beta$-blockade for preventing variceal bleeding: a case series. Ann Intern Med 2020;173:404-5.

69. Drucker DJ. The cardiovascular biology of glucagon-like peptide-1. Cell Metab 2016;24:15-30.

70. Simes BC, MacGregor GG. Sodium-glucose cotransporter-2 (SGLT2) inhibitors: a clinician's guide. Diabetes Metab Syndr Obes 2019;12:2125-36.

71. Wu JHY, Foote C, Blomster J, et al. Effects of sodium-glucose cotransporter-2 inhibitors on cardiovascular events, death, and major safety outcomes in adults with type 2 diabetes: a systematic review and meta-analysis. Lancet Diab \& Endocrinol 2016;4:411-9.

72. Kluger AY, Tecson KM, Lee AY, et al. Class effects of SGLT2 inhibitors on cardiorenal outcomes. Cardiovasc Diabetol 2019;18:99.

73. Cai X, Yang W, Gao X, et al. The association between the dosage of SGLT2 inhibitor and weight reduction in type 2 diabetes patients: a meta-analysis. Obesity (Silver Spring) 2018;26:70-80.

74. Raschi E, Parisotto M, Forcesi E, et al. Adverse events with sodium-glucose co-transporter-2 inhibitors: a global analysis of international spontaneous reporting systems. Nutr Metab Cardiovasc Dis 2017;27:1098-107.

75. Eriksson JW, Lundkvist P, Jansson PA, et al. Effects of dapagliflozin and n-3 carboxylic acids on non-alcoholic fatty liver disease in people with type 2 diabetes: a double-blind randomised placebo-controlled study. Diabetologia 2018;61:1923-34.

76. Kuchay MS, Krishan S, Mishra SK, et al. Effect of empagliflozin on liver fat in patients with type 2 diabetes and nonalcoholic fatty liver disease: a randomized controlled trial (E-LIFT Trial). Diabetes Care 2018;41:1801-8.

77. Inoue $\mathrm{M}$, Hayashi $\mathrm{A}$, Taguchi $\mathrm{T}$, et al. Effects of canagliflozin on body composition and hepatic fat content in type 2 diabetes patients with non-alcoholic fatty liver disease. J Diabetes Investig 2019;10:1004-11.

78. Kurinami N, Sugiyama S, Yoshida A, et al. Dapagliflozin significantly reduced liver fat accumulation associated with a decrease in abdominal subcutaneous fat in patients with inadequately controlled type 2 diabetes mellitus. Diabetes Res Clin Pract2018;142:254-63.

79. Shimizu M, Suzuki K, Kato K, et al. Evaluation of the effects of dapagliflozin, a sodium-glucose co-transporter-2 inhibitor, on hepatic steatosis and fibrosis using transient elastography in patients with type 2 diabetes and non-alcoholic fatty liver disease. Diabetes Obes Metab 2019;21:285-92.

80. Sattar N, Fitchett D, Hantel S, George JT, Zinman B. Empagliflozin is associated with improvements in liver enzymes potentially consistent with reductions in liver fat: results from randomised trials including the EMPA-REG OUTCOME® trial. Diabetologia 2018;61:2155-63.

81. Choi DH, Jung CH, Mok JO, Kim CH, Kang SK, Kim BY. Effect of dapagliflozin on alanine aminotransferase improvement in type 2 diabetes mellitus with non-alcoholic fatty liver disease. Endocrinol Metab (Seoul) 2018;33:387-94.

82. Bajaj HS, Brown RE, Bhullar L, Sohi N, Kalra S, Aronson R. SGLT2 inhibitors and incretin agents: associations with alanine aminotransferase activity in type 2 diabetes. Diabetes Metab 2018;44:493-9.

83. Akuta N, Watanabe C, Kawamura Y, et al. Effects of a sodium-glucose cotransporter 2 inhibitor in nonalcoholic fatty liver disease complicated by diabetes mellitus: preliminary prospective study based on serial liver biopsies. Hepatol Commun2017;1:46-52.

84. Wang H, Yang J, Chen X, Qiu F, Li J. Effects of sodium-glucose cotransporter 2 inhibitor monotherapy on weight changes in patients with type 2 diabetes mellitus: a bayesian network meta-analysis. Clin Ther 2019;41:322-34.e11.

85. Hung MH, Chen YL, Chen LJ, et al. Canagliflozin inhibits growth of hepatocellular carcinoma via blocking glucose-influx-induced $\beta$-catenin activation. Cell Death Dis 2019;10:420. 
86. Del Prato S. Heterogeneity of diabetes: heralding the era of precision medicine. Lancet Diab Endocrinol 2019;7:659-61.

87. Tricco AC, Antony J, Soobiah C, et al. Safety, effectiveness, and cost of dipeptidyl peptidase-4 inhibitors versus intermediate acting insulin for type 2 diabetes: protocol for a systematic review and network meta-analysis. Syst Rev 2013;2:47.

88. Liu ST, Su KQ, Zhang LH, Liu MH, Zhao WX. Hypoglycemic agents for non-alcoholic fatty liver disease with type 2 diabetes mellitus: a protocol for systematic review and network meta-analysis. Medicine (Baltimore) 2020;99:e21568.

89. MacIsaac RJ. Dulaglutide and insulin: how can the AWARD studies help guide clinical practice? Diabetes Ther 2020;11:1627-38.

90. Abdul-Ghani M, Migahid O, Megahed A, DeFronzo RA, Al-Ozairi E, Jayyousi A. Combination therapy with pioglitazone/exenatide improves beta-cell function and produces superior glycaemic control compared with basal/bolus insulin in poorly controlled type 2 diabetes: a 3-year follow-up of the Qatar study. Diabetes Obes Metab 2020; doi: 10.1111/dom.14153.

91. Vilsbøll T, Ekholm E, Johnsson E, Dronamraju N, Jabbour S, Lind M. Dapagliflozin plus saxagliptin add-on therapy compared with insulin in patients with type 2 diabetes poorly controlled by metformin with or without sulfonylurea therapy: a randomized clinical trial. Diabetes Care 2019;42:1464-72.

92. Zaccardi F, Dhalwani NN, Dales J, et al. Comparison of glucose-lowering agents after dual therapy failure in type 2 diabetes: a systematic review and network meta-analysis of randomized controlled trials. Diabetes Obes Metab 2018;20:985-97.

93. Cherney DZI, Dekkers CCJ, Barbour SJ, et al. Effects of the SGLT2 inhibitor dapagliflozin on proteinuria in non-diabetic patients with chronic kidney disease (DIAMOND): a randomised, double-blind, crossover trial. Lancet Diab Endocrinol 2020;8:582-93.

94. Papazafiropoulou AK, Melidonis A, Antonopoulos S. Effects of glucagon-like peptide-1 receptor agonists and sodium-glucose cotransporter 2 inhibitors on cardiorenal and metabolic outcomes in people without diabetes. Curr Pharm Des 2020; doi: 10.2174/138161 2826666200909142126.

95. Vincent RK, Williams DM, Evans M. A look to the future in non-alcoholic fatty liver disease: are glucagon-like peptide-1 analogues or sodium-glucose co-transporter-2 inhibitors the answer? Diabetes Obes Metab 2020; doi: 10.1111/dom.14196.

96. Brodosi L, Marchignoli F, Petroni ML, Marchesini G. NASH: a glance at the landscape of pharmacological treatment. Ann Hepatol 2016;15:673-81. 